\title{
Effect of 2,4-D and BAP on growth and chemical characteristics of bean sprouts (Vigna mungo L.)
}

\begin{abstract}
Bean sprouts or "tauge" is a popular vegetable among the Malaysians. Sprouts are rich in nutrients, easily digested, but are perishable and have a short life cycle. Relatively, standard sprouts have long, thin hypocotyls and long roots. Some farmers use plant growth regulators, such as the 2,4-dichlorophenoxyacetic acid (2,4-D) and 6-benzylaminopurine (BAP), to increase the hypocotyl thickness and inhibit bean sprout growth. Calcium makes the sprouts crispy, healthy and fresh. Thus, the objective of this study was to determine the efficiency of 2,4-D and BAP in influencing the growth of bean sprouts. Vigna mungo (black gram) seeds were used in this study. The bean seeds were treated with 2,4-D and BAP at 5, 10, 15 and 20 $\mathrm{mg} \mathrm{L}-1$, and each with the addition of $100 \mathrm{mg} \mathrm{L}-1$ calcium. The beans sprouts were analyzed for soluble solids concentration (SSC), $\mathrm{pH}$, titratable acidity and vitamin C. Physical characteristics, such as hypocotyl length, diameter and weight and root length, were determined. The experimental design was a randomized complete block design with a factorial arrangement of treatments. The results showed that seeds treated with 2,4-D and BAP produced significantly shorter sprout hypocotyl and roots length, thicker hypocotyls diameter and higher hypocotyls weight than sprouts from control (water treatment). Also, treated seeds produced sprouts with higher $\mathrm{pH}$, vitamin $\mathrm{C}$, SSC and titratable acidity compared to control. In conclusion, 2,4-D and BAP, produced desirable commercial characteristics of the bean sprouts.
\end{abstract}

Keyword: Plant growth regulator; Hypocotyl; Root; Cotyledon. 